\title{
ALGEBRAIC SPECIFICATIONS AND TRANSITION GRAPHS
}

\author{
Norman Y.Foo \\ Basser Department of Computer Science \\ University of Sydney
}

\author{
ABSTRACT \\ A simple connection between transition graphs and algebraic \\ specifications of data types is explained and its use in \\ extracting equations for data abstraction is illustrated.
}

\section{INTRODUCTION}

The algebraic specification to data abstraction has been advocated by several researchers including Guttag [G1], Isiskov and Zilles [II], and ADJ [AI]. In this approach a data type is abstracted by exhibiting a finite set of equations (axioms, identities, or laws) which hold between pairs of finite sequences of data operations. For a complete specification this set must be satisfied by the data operations and every conceivable equality between two operation sequences must be implied by it. Mathematically, as is argued in Guttag [GI] for example, an algebraic specification of a data type is really a hetexogeneous algebra in the sense of Birkoff and Iipson [B2] which captures the data type semantics. ADJ [A1] further argues that correct specifications must be initial objects, i.e. they must uniquely represent data types up to isomorphism; this was also advocated from a different viewpoint [F1] where the equivalent model-theoretic notion of categoricity was used.

A plausible strategy for the correct abstraction of data types could proceed as follows. Using some easily understood but quite possibly partial or semi-formal semantic description of a data type, one attempts to extract (operation sequence) equations consistent with the description. One could then use an automated initial-object or categoricity prover to verify that the extracted specification is correct. Efficient. realization of this strategy is a long-term goal.

This and related papers [NI] report some techniques which are proving to be helpful at different stages of our investigations. The main topic 
addressed here is a relationship between the state-transition graphs of data types and the equation sets which make up algebraic specifications. The semi-foxmal semantic description used is therefore the state behaviour of the data type. In almost all non-trivial data types the state space is unbounded, so a complete description of state behaviour is not possible in any pictorial representation of a transition graph. In some cases finitary descriptions are possible, e.g., using primitive recursive schemes, and these are in turn translatable into transition graphs. It will be seen that the relationship mentioned above can be used on partial representations of transition graphs to help extract equations (or suggest remedies when this proves to be difficult) for at least those data types where the operations are unary over a homogeneous domain. We will later indicate how this last restriction can be removed by re-interpreting standard transition graphs; the difficulties appear to be more notational than conceptual.

With these remarks the data types which will be primarily discussed in the sequel can be abstracted as homogeneous algebras as treated by Birkhoff [BI], or in a more model-theoretic vein by Tarski [TI], to which the reader is referxed for some results which are quoted here. The little graph theory and algebra that is presumed here can be found in seshu [S1] and Birkhoff [BI] respectively, and the automata-theoretic terminology is more than adequateIy covered by Booth [B3]. We also presume familiarity with the basic notions of algebraic specifications as presented in, say, the introductory portions of the reference cited at the beginning of this section. We introduce the notation required in the next section.

\section{GRAPH ALGEBRAS}

An algebra $Q=(A, \theta)$ is a pair where $A$ is the domain (carrier) of the algebra and $\theta$ is a finite set of operations on $A$. since we are restricting consideration to operations which are at most unary the arity (rank) of each operation $f$ in $\theta$ is 0 or 1 . The 0-arity operations are effectively constants in $A$ and correspond to fixed states of the data type, e.g., an initial 
state or an error state. The remaining unary operations represent the usual operations of the data type loperations of higher arity will be briefly considered when we discuss the removal of these restrictions). The kind of algebra which we use here to describe data types is a graph algebra deriving from the state-transition graph of the data type. Let $G=(Q, E)$ be such a graph, where the vertex set $Q$ is the extended state space of the data type, and $E$ the set of labelled directed edges represents the state transition function $\delta: \theta \times \quad Q \rightarrow Q$ ( $\theta$ being the operation set) in the usual way, viz., an edge labelled by $f$ goes from $q$ to $r$ if and only if $\delta(f, q)=r$. The extension to the state space of the data type mexely consists in adding a special element denoted by \# to it. This is done so that all operations can be uniformly represented in $G$ : a 0-ary operation $f_{0}$ will both name a state $f_{0}$ in $G$ as well as label an edge from \# to state $f_{0}$. G can be interpreted as an algebra in the obvious way. Every operation sequence on the data type corresponds to a directed path in $G$ and is a word (texm) of the algebra. An equation is a pair of $(\alpha, \beta)$ such words, normally written $\alpha=\beta$, denoting functional identity and interpreted in $G$ as path pairs which form circuits of a special kind - the e-circuits defined below.

Having explained what a graph algebra is we now review a completeness theorem proved by Birkhoff [BI] about syntactic inference rules which can be used to generate equations from a base set of equations. The formal language used in this prooftheory has the equality symbol and names for all operations in $\theta$. A term in this language is any legal string of operation symbols, i.e., if a 0 -ary name occurs it must only be rightmost in a string (composition is interpreted as left composition). In this latter case we call the terms type 1 , all other terms being of type 2. Formulas are of the form $\alpha=\beta$ where both $\alpha$ and $\beta$ are terms. A formula is of type 1 if both its terms are type 1 , and is type 2 otherwise. The meaning of formulas is given in $G$ by the following intexpretations. A type $I$ formula $\alpha=\beta$ is true in $G$ if $\delta(\alpha, \#)=\delta(\beta, \#)$; a type 2 formula $\alpha=\beta$ is true in $G$ if for all $\mathrm{q} \varepsilon Q \delta(\alpha, q)=\delta(\beta, q)$ or $\delta(\alpha, q)=\delta(\beta, \#)$. We write $G \neq \sigma$ to mean the formula $\sigma$ is true in $G . T(G)$ is the set $\{\sigma \mid G \neq \sigma\}$. 
$A$ basis for $T(G)$ is a subset $\sum$ of formulas such that $G * \sigma$ iff $G=\sum \neq \sigma$, i.e. $\Sigma$ logically implies $\sigma$ in $G$. This is a semantic concept whose syntactic counterpart was formalized and proved complete by Birkhoff in the above cited paper. The result states that $\Sigma$ is a basis for $T(G)$ iff $T(G)$ is the closure $\bar{\Sigma}$ of $\Sigma$ under the following derivation schema:

(i) $\Sigma \subset \bar{\Sigma}$ (ii) $\alpha=\alpha$ is in $\bar{\Sigma}$ for 211 terms $\alpha$

(iii) if $\alpha=\beta$ and $\beta=\gamma$ are in $\bar{\Sigma}$ then so is $\alpha=\gamma$

(iv) if $\alpha=\beta$ is in $\bar{\Sigma}$ then so is $f \alpha=f \beta$ for ald $f$ in $\theta$

(v) equals may be substituted for equals. If $\sigma$ is derivable from $\Sigma$ we write $\Sigma+\sigma$. The completeness of this schema is to say that $\Sigma$ is a basis for $T(G)$ iff $\Sigma \vdash T(G)$. An algebraic specification, as understood in the literature, exists for a data type with graph algebra $G$ precisely when $T(G)$ has a finite basis $\Sigma$.

Lerma 1

Rules (iii) and iv) can be replaced by rule (vi) below: (vi) $\alpha \mu \beta=\gamma$ and $\nu=\mu$ in $\bar{\Sigma}$ imply $\alpha \cup \beta=\gamma$ in $\bar{\Sigma}$

The proof of this lemma is easy and therefore omitted. We shall consider rules (i), (ii), (v) and (vi) to be the schema for obtaining $T(G)$. We wish to interpxet each of these rules into $G$ assuming that $\Sigma$ is a basis for $T(G)$. To do this easily we make use of the following definition.

\section{Definition}

An e-circuit at $q$ in $G$ is a pair $(q,\{\alpha, \beta\})$ such that $\delta(\alpha, q)=\delta(\beta, q)$ and a pseudo e-circuit is a pair $(q(\alpha, \beta)$ such that $\delta(\alpha, \#)=\delta(\beta, q)$ or vice versa.

The interpretation of the derivation rules depends on the type of equation, but in any case rule (i) interprets into $G$ as a set $\Sigma$ of e-circuits. The essential difference between type $I$ and type 2 interpretations is simply that type 1 equations $\alpha f_{0}=B g_{0}$, where the 0-ary operations have been written explicitly, denote e-circuits at \#, 
i.e., (\#, $\left.\left\{\alpha f_{0}, \beta g_{0}\right\}\right)$, while type 2 equations $\alpha=\beta$ really denote a global set of e-circuits $\{(q,\{\alpha, \beta\}) \mid q \varepsilon Q\}$. The latter however is not strictly mathematically correct and to be rigorous we should be really faking quotients - but this turns out to be unnecessary since a rule like (iii) simply asserts that in $G$ if $(q,\{\alpha, \beta\})$ and $(q,\{\beta, \gamma\})$ are e-circuits in $\bar{\Sigma}$ for every $q \varepsilon Q$ then $(q,\{\alpha, \gamma\})$ is also an e-circuit in $\bar{\Sigma}$ for every $q \varepsilon Q$. This last assertion is obviously true in $G$. Likewise the remaining rules are also true in $G$, as would have been expected from completeness.

The interesting rule is (vi). What this asserts is that a special kind of ring-sum of e-circuits is also an e-circuit. Recall [s1] that subsets of the directed circuits of directed graphs form an abelian group under the ring-sum addition of edges where these are counted modulo 2. In fact if one regards the integers modulo 2 as a field the system of circuits is a vector space. E-circuits are directed circuits of a particular form which in general are not closed under arbitrary ring-sums: a counterexample is suggested by Figure 1 . To be more precise suppose that in rule ( $v i) \mu=v$ is of type 1 . Then $\beta$ must be null in $\alpha \mu \beta=\gamma$, hence this equation is also of type 1 and may in fact be written $\alpha \mu=\gamma$. By the interpretation into $G$ the assumption of rule (vi) is that $(\#,\{\alpha \mu, \gamma\})$ and $(\#,\{\mu, \nu\})$ are e-circuits in $\bar{\Sigma}$, from which the conclusion is $(\#,\{\alpha \nu, \gamma\})$ is an e-circuit in $\bar{\Sigma}$. A pictorial representation of this is in Figure 2 showing the ring-sum interpretation of the rule.

The remaining cases are explained similarly and left to the reader. We say that an e-circuit $(q,\{\alpha, \beta\})$ translates to $r$ if $(r,\{\alpha, \beta\})$ is also an e-circuit. Restating the definition of $\Sigma$ being a finite basis for $T(G)$ in terms of the e-circuits of $G$ we may now say that a finite set $\Sigma$ of e-circuits generates (is a basis for) all e-circuits whenever $\Sigma$ decomposes into $\Sigma_{1} \Sigma_{2}$, the e-circuits in $\Sigma_{2}$ translate to all of $Q$, and every e-circuit in $G$ is a ring-sum of those in $\Sigma_{1}$ and the translates of those in $\Sigma_{2}$. The sets $\Sigma_{1}$ and $\Sigma_{2}$ correspond to equations of type 1 and 2 respectively. This observation is recorded as: 


\section{Theorem 1}

A data type has an algebraic specification iff its transition graph has e-circuits which are finitely generated. (1)

As it stands the result is not very useful because it says nothing about how one might search for such a basis in graphs. To remedy this we will now analyse operation equations (and hence e-circuits) to expose some simplifications. Specifically it will be shown that we need oniy consider the following forms of equations for any basis $\Sigma$ (a) $\alpha=\lambda$ where $\lambda$ is null (b) $\alpha=\beta$ where no proper suffixes $\alpha^{1}, \beta^{1}$ of $\alpha, \beta$ are such that $\alpha^{1}=\beta^{1}$ (c) $\alpha \mu=\mu$, where no proper suffix $\alpha^{1}$ of $\alpha$ is such that $\alpha^{1} \mu=\mu$, and (d) $\alpha \mu=\beta \mu$ where no proper suffixes $\alpha^{1}, \beta^{1}$ of $\alpha, \beta$ are such that $\alpha^{1} \mu=\beta^{1} \mu$. Interpreted as e-circuits these forms are shown in figure 3 .

Definition

An e-circuit $(q,\{\alpha, R\})$ such that for no proper suffixes $\alpha, \beta^{l}$ of $\alpha, \beta$ is $(q,\{\alpha, \beta\})$ an e-circuit is called simple. A path concatenated with a simple circuit is called p-simple.

The e-circuits denoted by equations in (b) above are simple. The loops in (a) are also simple. Those in (c) and (d) are paths concatenated respectively with loops and simple non-loops and therefore p-simple. Once this result is established the search for candidate e-circuits which can be used as basis sets $\Sigma_{1}$ and $\Sigma_{2}$ above will be considerably amplified. We proceed to do this.

By the index of an equation $\alpha=\beta$ we mean the following. Let $\alpha$ be $\mu \gamma$ and $\beta$ be $\nu \gamma$ where $\gamma$ is the longest common suffix of $\alpha$ and $\beta$, possibly null. Denoting the length of a word $\eta$ by $|\eta|$ the index of $\alpha=\beta$ is $|\gamma|+|\alpha|+|\beta|$. If $\gamma$ is null this is simply $|\alpha|+|\beta|$. The proof of the assertions above is by induction on the index of equations. Consider first an equation $\alpha=\beta$ of index 1. This must be of form (a) so our assertion is trivially

(1) In fact the graph-theoretic version of vector space bases for directed circuits can be shown to imply the Birkhoff completeness theorem and hence the two are equivalent. But we do not pursue this issue here. 
satisfied. Assume that the assertions are true for all equations of index less than $n$ and let $\alpha=\beta$ have index $n$. Either $\alpha$ and $\beta$ have a common suffix or they do not. Suppose they do not, and let $\alpha^{1}, \beta^{1}$ be the shortest suffixes of $\alpha, \beta$ respectively such that $\alpha^{1}=\beta^{1}$. If these are not proper suffixes then $\alpha=\beta$ is of form (b). If they are, we may replace $\alpha=\beta$ by the equations $\alpha^{1}=\beta^{1}$ and $\mu \alpha^{1}=v \alpha^{1}$ (where $\alpha$ is $\mu \alpha^{1}$ and $\beta$ is $\nu \beta^{1}$, , the first being of form (a) or (b), and the second has smaller index than $\alpha=\beta$ whence by the induction hypothesis it may be replaced by equations of forms (a) through (d). Suppose then that $\alpha$ and $\beta$ do have a common longest suffix $\gamma$ and let $\alpha$ be $\mu \gamma$, $\beta$ be $v \gamma$. Either $\mu, v$ have shortest proper suffixes $\mu_{1}, v_{1}$ such that $\mu_{1} \gamma=\nu_{1} \gamma$ or not. If not, $\alpha=\beta$ is of form (d). If $\mu_{1}$ and $\nu_{1}$ are proper then $\alpha=\beta$ may be replaced by the equations $\mu_{1} \gamma=\nu_{1} \gamma$ which is of form (c) or (d), and $\mu_{2} \mu_{1} \gamma=\nu_{2} \mu_{1} \gamma$ which is of smaller index than $\alpha=\beta$.

Two observations are made about the above proof. First, the replacements are valid via an appeal to the derivation rules. Second, the type of the equation $\alpha=\beta$ is preserved in the replacing equations.

Let us say that type 1 equations are irredundant in $\Sigma$ if $\Sigma_{1} \neq \emptyset$ and $\Sigma_{2}+\Sigma_{1}$. Type 1 equations are not irredundant in $\Sigma$ iff there exists an equivalent basis with only type 2 equations. Thus, if type 1 equations are irredundant in a given basis $\sum$ they must also be present in all bases. Otherwise there is a basis $\Sigma^{1}$ having only type 2 equations, and by the above construction $\Sigma^{1}$ may be assumed to be of the forms (a) through (d). Since $\Sigma i-\Sigma^{1}, \Sigma^{1}-\Sigma$ and - preserves equation type, $\Sigma$ is of type 2 , contradicting the assumption. We restate the preceding results in terms of e-circuits.

\section{Theorem 2}

The e-circuits of a transition graph are generated by basic sets consisting only of simple and p-simple e-circuits.

\section{Corollary}

A data type is algebraically specifiable iff its transition graph e-circuits are finitely generated by simple and p-simple e-circuits.

\section{Theorem 3}

Type 1 e-circuits (i.e. of the form $(\#,\{\alpha, \beta\})$ ) are irredundant in one basic set iff type 1 e-circuits are irredundant in all basic sets. 


\section{EXAMPLES AND APPLICATIONS}

Some simple examples and applications of the preceding characterizations are discussed in this section. A few cautionary remarks are in order. It should not be concluded that the use of transition graphs will yield easy and complete procedures for extracting equations for data types, but rather that they suggest what equations might be necessary and provide insight into the process of abstraction. Graph descriptions have obvious limitations if they are finite (in which case they will usually be only partial descriptions). Even when finitary descriptions are available things are not easy because the existence of algebraic specifications may not be decidable.

Theorem 4 .

If the state transition graph of a data type is described by primitive recursive fractions there is no algorithm to decide if the data type has an algebraic specification.

The proof of this will be merely outlined since the details are tedious. We show that if there is such an algorithm the Halting Problem for Turing machines is solvable, which is a contradiction. Without loss of generality we can modify the halting states to go into small loops. From Minsky [M2] we know that a Turing machine with a (say, given) input tape the next configuration (i.e. instantaneous description) function is primitive recursive. This function determines an autonomous transition graph in which we could trivially label every edge by a fixed operation symbol to define a graph algebra. Now, there are two possibilities - either the graph is an infinite path or else it is a path concatenated with a loop. In the first case there is no algebraic specification for the corresponding data type. In the second case there is an algebraic specification consisting of one type 1 equation of the form (c) in the previous section. So there is an algebraic specification iff (i) the machine loops around a modified halting state or (ii) the machine loops in other states. But for looping machines there are computable upper bounds on the number of steps which will reveal the nature of the loop. Hence an algorithm for deciding the existence of algebraic specifications in this case will yield one for deciaing halting. 
In [MI] Majster raised the question of hidden operations and algebraic specifications. In the present context hidden operations are new labelled edges in a transition graph which are consistent with the old edges. Consistency really means that it is not possible to derive a contradiction from the implied new set of equations and is equivalent to saying that the transition graph is deterministic.

\section{Definition}

A transition graph $G$ which has another graph $G^{1}$ as a subgraph is an inessential extension of it if the vertex sets of $G^{1}$ and $G$ are identical; it is a consistent extension if $G^{1}$ is deterministic (and hence $G$ too).

A data type may have a graph algebra without a finite basis and yet admit consistent inessential extensions which do have finite bases. Since we are only interested in reasonable extensions (although hidden states could be considered in an alternative treatment) we will simply say extension in the sense to mean consistent and inessential ones. As a good example of the preceding theory we consider a partial transition graph of the data type TOY-STACK which was proved by ADJ [ A2] to be not algebraically specifiable but which was shown by them to have an algebraically specifiable extension. TOY-STACK was intended as a simplification of a traversing stack data type introduced by Majster [ M1]. Its functional specification as given by $A D J$ is:

(1) $\operatorname{Error}($ ) = *

(2) Empty ( ) $=\langle 0,0\rangle$

(3) $\operatorname{Down}(*)=\operatorname{Push}(*)=*$

(4) Down $\langle p, s\rangle=$ if $p\rangle 0$ then $\langle p-1, s\rangle$ else *

(5) Push $\langle p, s\rangle=$ if $p=s$ then $\langle p+1, s+1\rangle$ else *

This does not have a finite basis, but on adding

(6) Shove $(*)=$ *

(7) Shove $\langle p, s\rangle=\langle p, s+1\rangle$

the new data-type has a finite basis. Using primes to denote the extension, Figure 4 shows partial transition graphs of these data types, To keep things simple we have abbreviated the operations and repeated the * state at various positions. We now argue for the non-existence of a finite basis of e-circuits for TOY-STACK from its transition graph to illustrate how such graphs could be used. 
It can be seen that $P D=\operatorname{Er}$ (it is perhaps more intuitive but somewhat misleading to write this as $P D=*$ ) denotes the only global set of e-circuits in the graph. These happen to be pseudo e-circuits. Now consider the set of e-circuits denoted by the equations $\left\{\mathrm{PD}^{\mathrm{n}} \mathrm{P}^{\mathrm{n}} \mathrm{Em}=\mathrm{DD}^{\mathrm{n}} \mathrm{P}^{\mathrm{n}}\right.$ Em $\mid n>I\}$. These are the downward paths concatenated with the horizontal ones ending in simple circuits in the figure. They are all independent in that none are generated by the others. Further, the pseudo e-circuits cannot be used in ring sums with the paths since this results in disconnection. Hence the e-circuits corresponding to these equations do not have a finite basis and TOY-STACK is not algebraically specifiable. By extending TOY-STACK to TOY-STACK ${ }^{1}$ with the introduction of the hidden operation "shove" the new data type was shown by ADJ to be algebraically specifiable. An examination of the transition graph of TOY-STACK ${ }^{1}$ reveals its increased regularity over that of TOX-STACK. This is to be expected since the graphs with finite bases are precisely those with a finite number of type 1 e-circuits and a finite number of type 2 e-circuits such that every e-circuit is the ring-sum of the former and (translates of) the latter.

To illustrate the process of extracting this information from typical graphs we use TOY-STACK ${ }^{1}$ as an example. Any such process using graph descriptions cannot, of course, be completely algorithmic on account of theorem 4, but it is not inconceivable that such extraction heuristics combined interactively with an automated initial object or categoricity prover could indeed yield a correct set of equations. It is convenient to tabulate the various rules in the heuristic as follows: (1) for constant states, i.e. those which are "named" by 0-ary operations, list the global pseudo e-circuits which lead into these states; (2) for all states, list the global simple and p-simple e-circuits; (3) other residual simple and p-simple e-circuits which are not ring-sums of (translates of) those in (1) and (2) are listed as type 1 e-circuits using paths from \# if necessary.

In looking for global e-circuits absorbing states can be ignored. A state is called an absorbing state in automata theory when no exit from it is possible; in such a case every pair of words $\{\alpha, \rho\}$ are suitable labels for e-circuits at that state. Applying these rules to ToY-STACK ${ }^{1}$ we extract the following equations. Since * is the only constant state 
and the only global paths that lead into it are named by the words PS and $\mathrm{PD}$, equations $\mathrm{PS}=\mathrm{Ex}$ and $\mathrm{PD}=\mathrm{Er}$ will suffice. This completes rule 1 . For the other states the simple e-circuit which is global is the one denoted by the equation SD = DS, depicted in the figure by the parallelograms. The right angled triangles $(e . g .\langle 0,0\rangle,\langle 1,1\rangle,\langle 0,1\rangle)$ apply precisely to states which are reached by a $P$ operation, and hence become global if they are denoted by the equation D P P = SP; these are in fact p-simple e-circuits. Next one may write down all the equations which say that * is an absorbing state, e.g., D Er = Er etc. This completes rule (2). The residual e-circuits which are not ring-sums of the preceding are those at \#. These are specified by the equations $D$ Em $=$ Er and $D$ P Em = S Em. This completes rule (3). In fact ADJ shows that this set of equations is categorical and hence correct for TOY-STACK ${ }^{1}$. We have produced here one more equation than was used by them, namely $\mathrm{ED}=\mathrm{Er}$ which turns out to be redundant, but this is not of great consequence in the light of our objectives.

Some additional remarks may be helpful. Since the graphical representation of state transitions is important for this heuristic, visual clues (or their automated pattern matching equivalents) are necessary. To this end let us recall concepts from classical automata theory [B3]. A homing sequence is a word which leads to a unique state from any arbitrary state. If we relax this definition to say that a homing sequence $\mu$ is a word which leads to a subset of states in which a certain e-circuit label $\{\alpha, \beta\}$ is global then $\alpha \mu=\beta \mu$ is an equation for the data type denoting p-simple e-circuits. Similar remarks hold for pseudo e-circuits. Hence what we are after in the applications of rules (1) through (3) above is really a (not necessarily disjoint) decomposition of the state space into homing sets identified by homing sequences, such that within each homing set the graph looks uniform with regard to its simple or pseudo e-circuits.

\section{CONCLUUSION}

We have presented a connection between transition graphs and algebraic specification of data types. Rules were given for the extraction of equations from transition graphs but we also indicated that even if the information contained in the transition graph is finitary these rules 
cannot be algorithmic. We believe that the techniques discussed here are useful aids in the abstraction of data types starting from intuitive state descriptions, and envisage that further development would combine automated versions of these with automated provers as software tools.

\section{REFERENCES}

[Al] ADJ (Goguen, Thatcher \& Wagner) - "An Initial Algebra Approach to the specification, Correctness and Implementation of Data Types". Current Trends in Programming Methodology, Vol.rV, Data structuring (Ed) R. Yeh, Prentice Hall, 1978.

[A2] ADJ (Thatcher, Wagner \& Wright) "Data Type Specification and the Power of Specification Techniques". Proceedings SIGACT 10th Annual stoc.

[B1] Birkhoff, G. "On the Structure of Abstract Algebras" Proc. Cambridge Phil. Soc. Vol. 31, part 4, Oct. 1935.

[B2] Birkhoff, G. \& Lipson, D. "Heterogeneous Algebras". J. Combinatorial Theory, 8, 1970.

[B3] Booth, T. "Sequential Machines and Automata Theory". Wiley, 1968.

[F1] Foo, N. \& Nolan, G. "Correctness of Algebraic Specifications for Data Structures ${ }^{\prime \prime}$ Aust. Univ. Computer Science Seminar, 1978.

[G1] Guttag, J. "The Specification and Epplication to Programming of Abstract Data Types". Univ. of Toronto, CSRG Tech. Report 59, sept. 1975 .

[II] Liskov, B. \& Zilles "Formal Specifications for Data Abstraction". Current Trends in Programing Methodology, Vol II, (ed) R. Yeh, Prentice Hall, 1977.

[MI] Majster, M. "Limits of Algebraic Specifications". SIGPLAN Notices, 12, 1977.

[M2] Minsky, M, "Computation: Finite and Infinite Machines" Prentice Hall, 1967.

[N1] Nolan, G. "DASIMI. A Practical Exercise in Data Abstraction". 
[SI] Seshu, S. \& Reed, M. "Linear Graphs and Electric Networks". Addison-Wesley 1961.

[Tl] Tarski, A. "Equational Logic and Equational Theories of Algebras". Contributions to Mathematical Logic (ed) Schutte, $\mathrm{K}$. North Holland, 1968. 